\title{
Effect of chin position on natural head orientation reproducibility
}

\author{
Mohammad-Hosein Ahangar-Atashi, Mojgan Kachoei
}

DDS, MSc Assistant Professor, Department of Orthodontics, Faculty of Dentistry, Tabriz University of Medical Sciences

Correspondence:

Department of Orthodontics,

Faculty of Dentistry,

Daneshgah St. Azadi St. Tabriz, Iran

dr_mojgan2004@yahoo.com

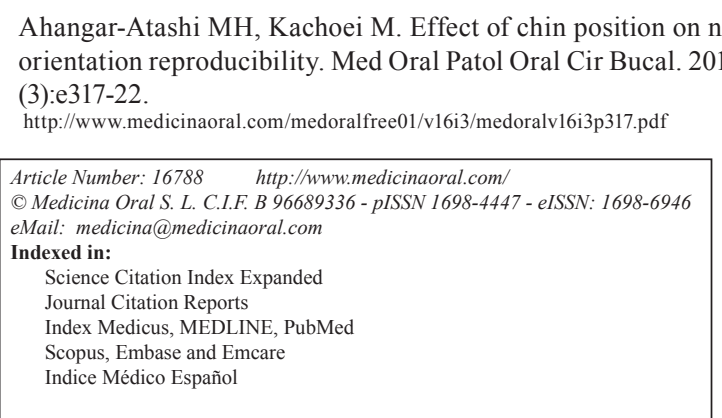

Received: 06/02/2010

Accepted: 26/08/2010

\begin{abstract}
Background and aims: Establishment of natural head position (NHP) in radiography or photography by estimation, which is called natural head orientation (NHO), has been recently advocated by various studies. This study aimed to determine the effect of chin position on the accuracy and reproducibility of NHO in a more realistic way.

Materials and Methods: NHP-based digital photographs of 33 adults with normal profile were modified using the computer software Supper-Goo and Photoshop technique to produce 99 profile images in three groups with normal, forward and backward chin positions. The reconstructed images were presented to three observers who rotated the images through a circular hole on a computer monitor. The horizontal angle was read on screen and recorded whenever they found the best horizontally-oriented face. This was performed at four intervals: T0: baseline; T1: after 5 minutes; T2: after one week; and T3: one month after baseline.

Results: After orientation, the mean orientation angles were $0.19^{\circ} \pm 1.39,0.20^{\circ} \pm 1.53$ and $-0.31^{\circ} \pm 1.38$ for the normal, forward and backward chin groups, respectively. T-test did not show any significant differences in NHO and NHP among the three groups of chin position: P values of $0.17,0.19$, and 0.26 for normal, forward, and backward chin positions, respectively. However, one-way ANOVA demonstrated significant differences in the NHO between the normal and backward, and backward and forward chin groups. The obtained reproducibility rate of NHO calculated via Med-Calc 8.1 online software for $\mathrm{T}_{0}, \mathrm{~T}_{1}, \mathrm{~T}_{2}$ and $\mathrm{T}_{3}$ intervals was more than $60 \%$, which is regarded significant in medical studies.

Conclusion: Natural head position could be created with great reproducibility through orientation of profile images in different chin positions. NHO is under the influence of chin position; however, this influence is not clinically significant.
\end{abstract}

Key words: Chin position, natural head orientation, reproducibility. 


\section{Introduction}

Natural head position (NHP) provides a stable position of the external reference planes for better analysis of lateral cephalograms of patients since it has high reproducibility rate (only 1 or 2 degrees) $(1,2)$.

Despite the fact that NHP has attracted a lot of attention in recent decades, several problems regarding the timeconsuming NHP registration technique have been reported, such as trouble orientating the head in children, tendency of patients with Class II or Class III malocclusions to mask their original jaw mal-relationships, and the need for well-trained technicians and speciallyequipped radiographic rooms (metal chain, mirror ...) (3-7).

The idea of imitating NHP by estimation has led to a new technique called natural head orientation (NHO). This is an easy way of providing a standard profile view similar to NHP, which eliminates difficulties related to this method (8). A significant correlation has been found between registered NHP and estimated NHP, both on photographs and on cephalograms (8).

Estimated natural head position or natural head orientation (NHO) has been proposed as a preferred reference position for assessing facial morphology (9).

The idea of head orientation for better evaluation dates back to 1982 when anatomists and anthropologists in Frankfort congress propounded the Frankfort plane as a reference when the skull is orientated parallel to horizon. However, several studies have suggested that the Frankfort plane is not always as horizontal and is deviated from true horizontal line based on NHP in many cases (10-12).

Viazis, following previous investigators, used the optic plane for cephalometric analysis. This plane is a bisecting line of the orbital angle passing through the pupils of the eyes (13).

Estimation of abnormal head postures has been recently proposed by ophthalmologists for clinical assessment of patients with strabismus and nystagmus (14).

Houston applied the average mean difference between SN (sella -nasion) line and true horizontal line to orientate images, by which he converted the conventional lateral cephalograms to NHP and obtained cephalometric norms using extra-cranial reference lines $(15,16)$.

Recently research centers, which have collected a lot of invaluable records of lateral cephalograms in their archives for a long period, have attempted to convert the conventional cephalograms to NHP. In this technique a circular hole is created by cutting off the center of a rectangular frame, and then the image is rotated inside the circle to obtain a naturally-oriented profile image. After estimation is completed, the true horizontal and true vertical lines are determined using the outer vertical and horizontal lines of the frame (3).

Estimated natural head position (NHO) has been report- ed to be as reliable and reproducible as registered natural head position (NHP). In Lundstrom's studies, the mean difference between NHP and NHO was less than $1.4^{\circ}$ and the standard error for $\mathrm{NHO}$ was less than that of NHP $\left(2.1^{\circ}\right.$ for NHO and $4.1^{\circ}$ for NHP) $(3,7,17,18)$.

As NHO is subjectively defined by the observer, some studies have demonstrated a negative effect of chin position in determining NHO through the observer's judgment. According to these studies, observers tend to rotate the face upward and forward in cases with retruded chin position, whereas in cases with protrusion of the chin, they usually adjust the head downward and backward $(3,9,19)$.

The aim of the present study was to evaluate the effect of chin protrusion or retrusion on accuracy and reproducibility of NHO in 3 groups with normal, protruded and retruded chin positions evaluated by three observers at four intervals. A new method of evaluation was designed for orientation of images using computer monitors, and large samples were analyzed.

\section{Materials and Methods}

This study was a descriptive analytical one carried out at Tabriz University of Medical Sciences in 2006.

The sample size was calculated to be 30 according to the formula $\mathrm{p}_{1}=0.8, \mathrm{p}_{2}=0.75, \alpha=0.05, \mathrm{~d}=0.09$ and a power of $80 \%$. This sample size was increased to 33 to increase the power of the study. The samples included 19 boys and 14 girls with an age range of 15-20 years, who were selected from the patients of a private clinic in Tabriz. Informed written consent was obtained from all the subjects and The Ethics Committee approved the study.

Two orthodontists and one general practitioner evaluated the subjects clinically so that all had straight and proportionate profiles with no chin deficiency or protrusion.

Digital photographs of profile views of the subjects were taken in NHP using a digital camera (Kolpix 8700, Nikon) by an expert orthodontist. An adjustable device with a frame and a metal chain was designed for proper adjustment of subjects' head. The subjects sat on a chair looking forward into their eyes in a mirror in front and a chain was hung with a distance of 5-10 $\mathrm{mm}$ from the nose bisecting the face on the frontal view.

All the photographs were transferred to a computer and an imaging software (Super Goo 1998, Meta Creations Corp) was used to wrap and produce profile images of backward and forward chins, which has similarities to the method used by Halazonetis for making changes in chin positions in the subjects (9). As a result of this procedure, 99 images including 33 normal, 33 backward and 33 forward chins were obtained (Fig. 1).

The images with poor quality or an improperly reconstructed chin were omitted. An image software program 


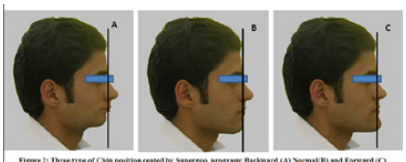

Fig. 1. Three types of chin positions ceated by Supergoo software; Backward (A), Normal (B) and Forward (C).

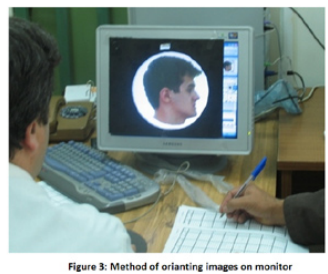

Fig. 2. Method of orientating the images on the monitor.

(Photoshop 7.0, Adobe, USA) was used to horizontally fit all the lateral photographs to a 15 -inch monitor $(56 \%$ magnification) by superimposing the chain on the vertical line of Photoshop. All the images were changed to a round shape with a diameter of 19.5 millimeters and the background of the images, including the image of the chain, was rubbed off from the photographs.

Method of orientation: A dark-colored rectangular cardboard with the same size as the monitor was fitted on the screen of the monitor with a 19.5-millimeter circular hole at its center. It covered the screen completely except for a narrow bar in front of the images to use the required menu and see the mouse and a small opening to read the rotation angle of the images. This small opening was only accessible by indirectly looking from the above after completion of each orientation (Fig. 2).

The round-shaped images were put in the circular hole with the same size and were rotated by three observers, including two orthodontists and a general practitioner. As the computer assistant presented the images of different chin positions randomly, the observers were unaware of the presented case. The assistant also presented the images with the angle of rotation set randomly before orientation; therefore, rotations of the images began from a different point other than the zero point. The observers positioned themselves one meter away from the monitor and used the mouse to rotate the images upward or downward until they found the best horizontal position relative to the rectangular frame of the monitor.

When the observers expressed the completion of orientation, the assistant read and recorded the rotational angles (the angle between NHP and NHO true horizontal plane). Positive measurements signified that the head was positioned in a flexed position and negative measurements showed an extended position.
The orientation of the images for normal, backward and forward chins was performed at four intervals: $\mathrm{T}_{0}$ : baseline; $\mathrm{T}_{1}$ : after 5 minutes; $\mathrm{T}_{2}$ : after one week; and $\mathrm{T}_{3}$ : one month after baseline.

After orientation of all the images, the samples were regrouped again to have accurate and different chin positions. The distance between soft tissue Pogonion to a line from soft tissue Nasion parallel to the true vertical line (0-degree meridian) (3) was measured in millimeters using the ruler of Photoshop program and also by digital calipers outside the monitor screen. As a result, the three groups of profiles were listed as follows: Normal group (original profile images, $-2 \leq$ pog'-N-prependicular $\leq+2$ ); the backward group in which the Pg' was more than 2 millimeters behind the N'-Vertical (pog'$\mathrm{N}$-prependicular $<-2$ ), and the forward group in which the Pg' was more than 2 millimeters forward than the N'-Vertical (pog'-N-perpendicular $>+2$ ).

The data regarding orientation process for the three groups at different intervals were analyzed using SPSS 17.0 software and descriptive analysis (mean and SD) was calculated for different groups and observers at different orientation intervals.

Kolmogorov-Smirnov test was used to confirm normal data distribution.

One sample t-test was used to compare the means of orientation angles of three chin position groups with NHP (zero), and one-way ANOVA was used to compare the means of orientation angles between the observers and among the three groups of chin positions.

A $\mathrm{P}$ value of $<0.05$ was considered significant. A post hoc Tukey test was used in the case of significance. The estimation of all the images in NHP was repeated by the three observers at four different intervals of $\mathrm{T}_{0}$, $\mathrm{T}_{1}, \mathrm{~T}_{2}$ and $\mathrm{T}_{3}$ and the reproducibility of natural head orientation (NHO) for the normal, backward and forward chin groups was calculated using Medcalc 8.1 online software.

\section{Results}

The means and standard deviations for the distance between Pog and N-perpndicular in the three normal, backward and forward chin groups were $-0.31 \pm 1.37$ $\mathrm{mm},-8.59 \pm 2.94 \mathrm{~mm}$, and $+5.34 \pm 2.34 \mathrm{~mm}$, respectively.

The error in estimation of the NHO for the interval between the first and last measurements (T0 and T3) was assessed according to Houston (15) using the formula:

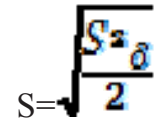

where $\mathrm{S}$ is the standard deviation of different measurements at $\mathrm{T} 0$ and $\mathrm{T} 3$. The errors were $1.49^{\circ}$, $1.30^{\circ}$ and $0.47^{\circ}$ for the first, second and third observers, respectively.

Descriptive analysis of mean estimation angles at dif- 
Table 1. Descriptive analysis of NHO assessed by three observers.

\begin{tabular}{|c|c|c|c|c|c|c|c|c|c|c|}
\hline \multirow{2}{*}{$\begin{array}{l}\text { Intervals of } \\
\text { Estimation }\end{array}$} & \multirow{2}{*}{$\mathbf{N}$} & \multicolumn{3}{|c|}{ Backward chin position } & \multicolumn{3}{|c|}{ Normal chin position } & \multicolumn{3}{|c|}{ Forward chin position } \\
\hline & & Mean & SD & Range & Mean & SD & Range & Mean & SD & Range \\
\hline T0 & 99 & -0.19 & 1.80 & 11.20 & 0.24 & 1.85 & 10.00 & 0.34 & 2.11 & 12.50 \\
\hline $\mathrm{T} 1$ & 99 & -0.27 & 1.85 & 11.30 & 0.12 & 2.04 & 11.60 & 0.22 & 2.09 & 12.80 \\
\hline $\mathrm{T} 2$ & 99 & -0.26 & 1.80 & 8.20 & 0.32 & 1.83 & 10.00 & 0.13 & 2.11 & 11.90 \\
\hline $\mathrm{T} 3$ & 99 & -0.53 & 1.84 & 9.00 & 0.10 & 1.69 & 8.30 & 0.11 & 1.94 & 10.40 \\
\hline Sum & 396 & -0.31 & 1.38 & 7.83 & 0.19 & 1.39 & 6.78 & 0.20 & 1.52 & 7.18 \\
\hline
\end{tabular}

ferent intervals separately and in the aggregate regarding the chin position and regardless of the observers are shown in the table 1.

One-sample t-test did not demonstrate any significant differences between overall averages of NHO in different chin positions and NHP (zero) (P values of 0.17, 0.19, and 026 for normal, forward, and backward chin positions, respectively).

One-way ANOVA demonstrated the effect of chin position on NHO. A post hoc Tukey test showed that the difference between the normal and forward chin groups was not significant $(\mathrm{P}=0.98)$ whereas it was significant between the normal and backward $(\mathrm{P}=0.032)$ and between the forward and backward groups $(\mathrm{P}=0.028)$.

Regarding mean NHO difference between the observers, one-way ANOVA did not demonstrate any significant differences among the three observers in the normal $(\mathrm{P}=0,061)$ and forward $(\mathrm{P}=0.059)$ groups but in the backward group there was a significant difference between the observers ( $\mathrm{P}=0.0001)$ (Fig. 3).
According to Medcalc 8.1 online software, the reproducibility for NHO for the three observers at the four different intervals used in this study was more than $60 \%$, which has been considered significant in medical trials (Table 2).

\section{Discussion}

Some disadvantages of NHP have been described in literature. It was shown by Cook, Moorrees, and Lundstrom for two repeated cephalograms that the NHP is not a stable position. Lundstrom showed that the reproducibility of NHP is less than NHO. He believed that radiography equipment influences the internal physiologic responses $(1,6,7)$. This is more significant for children who have trouble adjusting the head (3).

Claman believed that the type of malocclusion could also affect NHP. Patients with Class II and Class III malocclusion hold their head in a way to mask the problems related to their malocclusion and to improve their

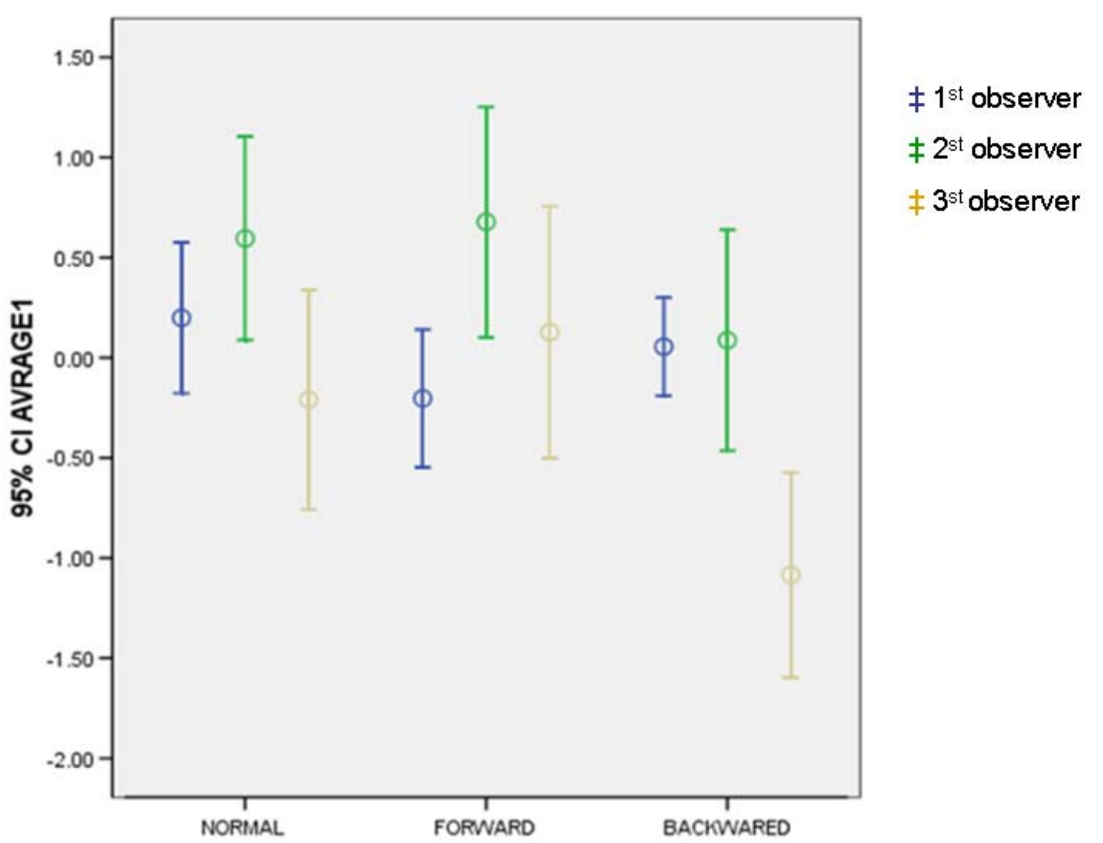

Fig. 3. Error bar diagram comparing mean $\mathrm{NHO}$ of the normal, forward and backward chin groups between the three observers. 
Table 2. The reproducibility of NHO for the three observers at four different intervals.

\begin{tabular}{|c|c|c|c|c|}
\hline Observers & $\begin{array}{c}\text { The base point } \\
\text { (T0) }\end{array}$ & $\begin{array}{c}\text { After five minutes } \\
\text { (T1) }\end{array}$ & $\begin{array}{c}\text { After one week } \\
\text { (T2) }\end{array}$ & $\begin{array}{c}\text { After one month } \\
\text { (T3) }\end{array}$ \\
\hline The $\mathbf{1}^{\text {st }}$ observer & $81 \%$ & $83 \%$ & $87 \%$ & $83 \%$ \\
\hline The $\mathbf{2}^{\text {nd }}$ observer & $79 \%$ & $80 \%$ & $78 \%$ & $81 \%$ \\
\hline The $\mathbf{3}^{\text {rd }}$ observer & $79 \%$ & $80 \%$ & $86 \%$ & $82.5 \%$ \\
\hline The sum & $81 \%$ & $84 \%$ & $78 \%$ & $69 \%$ \\
\hline
\end{tabular}

appearance (4). It may be the reason why the technique error in NHP is higher than that in NHO (17).

Natural head orientation (NHO) has been suggested as a way to minimize errors introduced by the physiologic responses during registration (3), though there are a few studies on the negative effects of the chin and profile morphology on observer's judgments in this method. In the case of Class II patients with backward chin position, for example, the observers tend to rotate the face upward and forward whereas in Class III, forward chin cases, they usually put the head downward and backward $(3,9)$.

In this study the data obtained from four different intervals by three observers did not demonstrate any statistical differences in NHP and NHO between the three chin groups. These results are different from the findings of Lundstrom et al. and Halazonetis $(3,9)$. The differences might be attributed to some factors such as different photographic or radiographic techniques, the method of obtaining NHO images (manual or computerized), the number, gender and experience of observers and factors related to subject selection (size, age or type of the profile of the samples).

In this study, three groups consisting of 33 photographs in three chin positions were oriented by three observers at four intervals: T0, T1, T2 and T3; therefore, the results were derived from 1188 orientations. This is the highest repetition rate of image orientation among studies found in literature.

The mean differences between registered and estimated natural head positions for normal, backward and forward chin groups were $0.19 \pm 1.39,-0.31 \pm 1.39$, and $0.20 \pm 1.53$, which circle around $+1^{\circ}$ (in 396 times of orientations for each). This finding is almost consistent with Lundstrom's finding $\left(+1.4^{\circ}\right)$ and confirms his idea that estimated natural head position (NHO) can be as accurate as registered natural head position (NHP) (3). According to the results of this study, short-term (T1, $\mathrm{T} 2$ ) and long-term reproducibility rates (T3) of NHO for all the subjects are as high as of that for NHP regardless of chin position. This finding is consistent with the results of studies carried out by Lundstrom et al. $(3,7)$, and Lundstrom and Lundstrom (10) but is not consistent with the results of studies carried out by Jiang et al. (8) and Halazonetis (9).

Some particular aspects of this study are described as follows:

1. Offering the images randomly to the observers and allocating the images in three groups of chin position (based on the distance of Pogonion to a line perpendicular to $\mathrm{N}$ ) after completion of the orientation diminishes the case selection judgment biases.

2. Wrapping only the chin on the images renders the mid-face and upper-face unchanged so the results could be dependent only on chin alteration and create really normal, forward and backward chin cases.

3. The monitor makes it possible to evaluate the profile of the cases with similar distance and size of the face in the same manner as in the clinic.

Although not statistically significant, in this study, the positive sign in normal and forward chin groups indicates a little downward and backward rotation of the head whereas the negative sign in backward group shows a minor forward and upward rotation of the head in photographs registered in NHO than in NHP. Lundstrom's results, in which he found a more extended head position in photographs registered in NHO than those registered in NHP, are the same as the findings of this study for backward chin group but are not consistent with the findings in the normal and forward chin groups (10). The differences between the two studies might be attributed to the lack of distinctions in the type of the profile in the photographs taken in Lundstrom's study.

\section{Conclusion}

Natural head position could be created with great reproducibility through orientation of profile images in different chin positions.

$\mathrm{NHO}$ is under the influence of chin position; tendencies for head extension in Class II patients and for head flexion in Cass III patients exist in the clinicians for orientation of the images; however, there are no statistically significant differences between NHP and NHO in different chin positions. 


\section{References}

1. Cooke MS, Wei SH. A summary five-factor cephalometric analysis based on natural head posture and the true horizontal. Am J Orthod Dentofacial Orthop. 1988;93:213-23.

2. Peng L, Cooke MS. Fifteen-year reproducibility of natural head posture: A longitudinal study. Am J Orthod Dentofacial Orthop. 1999;116:82-5.

3. Lundström A, Forsberg CM, Westergren H, Lundström F. A comparison between estimated and registered natural head posture. Eur J Orthod. 1991;13:59-64.

4. Claman L, Patton D, Rashid R. Standardized portrait photography for dental patients. Am J Orthod Dentofacial Orthop. 1990;98:197205

5. Fernández-Riveiro P, Smyth-Chamosa E, Suárez-Quintanilla D, Suárez-Cunqueiro M. Angular photogrammetric analysis of the soft tissue facial profile. Eur J Orthod. 2003;25:393-9.

6. Moorrees CF, Kent RL Jr, Efstratiadis SS, Reed RB. [Analysis of the components of facial growth by the structural superimposition of profile teleradiographs]. Schweiz Monatsschr Zahnmed. 1986;96:1513-28

7. Lundström A, Forsberg CM, Peck S, McWilliam J. A proportional analysis of the soft tissue facial profile in young adults with normal occlusion. Angle Orthod. 1992;62:127-33.

8. Jiang J, Xu T, Lin J. The relationship between estimated and registered natural head position. Angle Orthod. 2007;77:1019-24.

9. Halazonetis DJ. Estimated natural head position and facial morphology. Am J Orthod Dentofacial Orthop. 2002;121:364-8.

10. Lundström A, Lundström F. The Frankfort horizontal as a basis for cephalometric analysis. Am J Orthod Dentofacial Orthop. 1995; 107:537-40.

11. Petricevic N, Celebic A, Celic R, Baucic-Bozic M. Natural head position and inclination of craniofacial planes. Int J Prosthodont. 2006;19:279-80.

12. Barbera AL, Sampson WJ, Townsend GC. An evaluation of head position and craniofacial reference line variation. Homo. 2009;60:128.

13. Viazis AD. A cephalometric analysis based on natural head position. J Clin Orthod. 1991;25:172-81.

14. Kim DS, Coats DK, McCreery KM, Paysse EA, Wilhelmus KR. Accuracy of clinical estimation of abnormal head postures. Binocul Vis Strabismus Q. 2004;19:21-4.

15. Houston WJ. Bases for the analysis of cephalometric radiographs: intracranial reference structures or natural head position. Proc Finn Dent Soc. 1991;87:43-9.

16. Lundström A, Cooke MS. Proportional analysis of the facial profile in natural head position in Caucasian and Chinese children. $\mathrm{Br}$ J Orthod. 1991;18:43-9.

17. Bass NM. The aesthetic analysis of the face. Eur J Orthod. 1991;13:343-50.

18. Lundström A, Lundström F, Lebret LM, Moorrees CF. Natural head position and natural head orientation: basic considerations in cephalometric analysis and research. Eur J Orthod. 1995;17:111-20.

19. Halazonetis DJ. Morphometric evaluation of soft-tissue profile shape. Am J Orthod Dentofacial Orthop. 2007;131:481-9.

\section{Acknowledgement}

The authors would like to express their gratitude to Tabriz University of Medical Sciences for scientific and financial support of this study. 